\title{
Serum procalcitonin levels associate with Clostridioides difficile infection in patients with inflammatory bowel disease
}

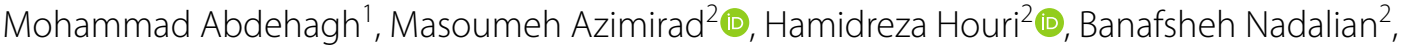 \\ Fahimeh Azimirad ${ }^{2}$, Meysam Olfatifar ${ }^{3}$, Ome Kolsoum Nasir Shoeibi ${ }^{1}$, Abbas Yadegar2* ${ }^{2 *}$, \\ Shabnam Shahrokh ${ }^{1 *} \mathbb{D}$, Mehran Mahdavi Roshan ${ }^{1}$, Hamid Asadzadeh Aghdaei ${ }^{3}$ and Mohammad Reza Zali ${ }^{1}$
}

\begin{abstract}
Background: Clostridioides difficile infection (CDI) is a major cause of morbidity among patients with inflammatory bowel disease (IBD). Diagnostic biomarkers for early detection of CDI are needed in clinical practice. The relationship between serum procalcitonin and CDI in IBD patients has not been investigated so far. Therefore, we aimed to evaluate the usefulness of measuring serum procalcitonin level to detect CDI in patients with the flare of IBD.
\end{abstract}

Methods: One hundred twenty patients with IBD were enrolled in this study. Bacterial identification was performed using standard microbiological and molecular methods. The serum procalcitonin levels were measured in all patients. Receiver operating characteristic (ROC) curve analysis was applied to assess the value of procalcitonin for the prediction of CDI among IBD patients.

Results: The median serum procalcitonin level was significantly increased in IBD patients with CDI compared to nonCDI IBD patients $(0.69 \mathrm{ng} / \mathrm{mL}$ vs $0.32 \mathrm{ng} / \mathrm{mL})$. In univariate analysis, $\log _{10}$ procalcitonin was associated with CDI (OR $2.81,95 \% \mathrm{Cl} 1.54-4.09$, P-value < 0.001 ). Procalcitonin $1.1 \mathrm{ng} / \mathrm{mL}$ was $85 \%$ sensitive and $88 \%$ specific for the prediction of CDI. In the multivariable model including the covariates $\log _{10}$ procalcitonin, age, hospitalization, type of IBD, duration of the disease, and antibiotic usage, procalcitonin showed a robust association with CDI (OR 4.59, 95\% Cl 2.49$6.70, P$-value $<0.001$ ). An elevated procalcitonin level was associated with the presence of CDI among IBD patients.

Conclusions: Our results indicate that procalcitonin level can be a good candidate biomarker for assessing the CDI in IBD patients. Further studies are required to decipher whether procalcitonin can predict CDI therapy or its recurrence.

Keywords: Clostridioides difficile infection, Inflammatory bowel disease, Procalcitonin level, IBD flare, Predictive biomarker, CD, ROC curve analysis

*Correspondence: a.yadegar@sbmu.ac.ir; shabnamshahrokh@gmail.com ${ }^{1}$ Gastroenterology and Liver Diseases Research Center, Research Institute for Gastroenterology and Liver Diseases, Shahid Beheshti University of Medical Sciences, Tehran, Iran

2 Foodborne and Waterborne Diseases Research Center, Research Institute for Gastroenterology and Liver Diseases, Shahid Beheshti University of Medical Sciences, Tehran, Iran

Full list of author information is available at the end of the article

\begin{abstract}
Background
Inflammatory bowel disease (IBD), including Crohn's disease (CD) and ulcerative colitis (UC), is regarded as a heterogeneous chronic and relapsing inflammatory condition of the gastrointestinal (GI) tract, with an unknown etiology and unpredictable clinical course [1]. Flare-up episodes of IBD are usually characterized by abdominal pain, diarrhea, rectal bleeding, fever, and weight loss [2]. Although specific etiological factors in the development
\end{abstract}


of IBD have not yet been completely elucidated, the current evidence points to a multifactorial origin, including predisposing genetic background, defects in local and systemic immune responses, the composition of the gut microbiota, and external environment $[3,4]$.

Although the occurrence of disease flares in IBD can be due to disease aggravation itself, it has been established that a bacterial infection with pathogenic organisms has a critical role in the deterioration and exacerbation of IBD [5]. Moreover, discriminating between disease flares and enteric infections in IBD patients is often problematic due to the similar clinical manifestations and laboratory findings [6]. Therefore, accurate early detection of enteric infections among IBD patients can prevent infection-related flares and severe complications such as bacteremia and sepsis. Importantly, the early detection of infections in IBD patients requiring immunosuppression, in which the infectious complications are facilitated in this population, has significant impact on the disease management and prognosis $[7,8]$. There is clear evidence that concurrent infections with opportunistic intestinal pathogens are increasingly recognized due to immunosuppressive therapy in IBD patients $[9,10]$. Apparently, patients with IBD appear to be at higher risk of severe Clostridioides difficile infection (CDI) than healthy people $[11,12]$.

The erythrocyte sedimentation rate (ESR), white blood cells (WBCs) count and C-reactive protein (CRP) level could be changed during enteric infections in IBD patients; however, these blood indexes may be elevated not only in infectious conditions but also during IBD flare-up [13, 14]. Recently, procalcitonin has been suggested as a novel biomarker for microbial infection in IBD. Procalcitonin is the precursor of calcitonin, an essential hormone produced in response to high levels of calcium in the bloodstream and involved in calcium homeostasis [15]. Serum procalcitonin is an excellent predictive factor for differentiating acute bacterial inflammatory conditions, however, it is not elevated by the inflammatory conditions of IBD itself. Literature review indicates that elevated procalcitonin levels could reflect the presence of many infective complications in IBD, including bacterial gastroenteritis and enterocolitis, postsurgical infection, intraabdominal abscess, and sepsis [16]. Furthermore, contrasted to CRP, the level of procalcitonin has been described to be unaffected during immunosuppression therapy in patients with IBD [17]. Hence, all these could be potentially taking advantage of procalcitonin to be applied as a biomarker for detection of bacterial infections for patients with IBD on immunosuppressive treatment. However, there are some unanswered questions in the efficacy of procalcitonin and CRP measurement in IBD flare patients with concomitant
CDI. This study, therefore, was conducted to explore the efficacy of serum procalcitonin measurement to detect CDI among patients with the flare of IBD.

\section{Materials and methods \\ Patients and sample collection}

Blood samples, colonic biopsies, and stool specimens were collected from 120 patients with IBD who were referred for colonoscopy at Research Institute for Gastroenterology and Liver Diseases in Tehran. Diagnosis of IBD was considered based on clinical, radiological, endoscopic, and pathological criteria, as described previously [18]. Patients were submitted to general interviews on the day of admission. A questionnaire, containing demographic data, medication, and clinical features was filled for all the patients. In our study, full remission was defined based on clinical remission and biochemical markers within normal limits. Accordingly, clinical remission was defined as a Crohn's disease activity index $(\mathrm{CDAI}) \leq 150$ for $\mathrm{CD}$ patients and Mayo Clinic score $\leq 2$ for UC patients, respectively [19, 20]. Biochemical remission in our evaluation was defined as a normal CRP $(\leq 5 \mathrm{mg} / \mathrm{L})$ and fecal calprotectin $(\leq 150 \mu \mathrm{g} / \mathrm{g}$ stool) [21, 22]. In this study, a CDI case is defined as a case of diarrhea (i.e., unformed stool that conforms to the shape of a specimen collection container) that the stool sample yields a positive culture result for a toxin-producing $C$. difficile organism without other known etiologies [23]. Subjects who had received $>48 \mathrm{~h}$ of antibiotics or were clinically indicated to be infected with other enteric bacterial and/or viral pathogens, such as Salmonella, Shigella, Campylobacter, Aeromonas, pathogenic Vibrio species and Escherichia coli strains, and active cytomegalovirus (CMV) infection were excluded. This study was approved by the Institutional Ethical Review Committee of Research Institute for Gastroenterology and Liver Diseases at Shahid Beheshti University of Medical Sciences (Project No. IR.SBMU.RIGLD.REC.1398.041). All experiments were performed in accordance with the relevant guidelines and regulations recommended by the Research Institute for Gastroenterology and Liver Diseases. Informed consent was obtained from all subjects and/or their legal guardians before sample collection.

\section{Bacterial culture and isolation}

For isolation of $C$. difficile, approximately $1 \mathrm{~g}$ of freshly stool specimens was treated with an equal volume of ethanol, vortexed, and held at room temperature for $2 \mathrm{~min}$. Homogenized stool specimens were cultured on cycloserine-cefoxitin-fructose agar (CCFA) (Mast Group Ltd., Merseyside, UK) supplemented with 7\% sheep blood. In addition, colonic biopsies were transported to the laboratory in thioglycolate broth (Merck, Darmstadt, Germany) 
and homogenized with a tissue grinder. For identification of $C$. difficile, a hundred microliter of each homogenized specimen was cultured on CCFA. The cultured plates were incubated under anaerobic conditions $\left(85 \% \mathrm{~N}_{2}\right.$, $10 \% \mathrm{CO}_{2}$, and $5 \% \mathrm{H}_{2}$ ) generated using Anoxomat ${ }^{\circledR} \mathrm{Gas}$ Exchange System (Mart Microbiology BV, Lichtenvoorde, Netherlands) at $37{ }^{\circ} \mathrm{C}$ for $48-72 \mathrm{~h}$. Identification of suspected colonies was performed by standard biochemical tests. Additionally, molecular detection of $C$. difficile was carried out using amplification of the $16 \mathrm{~S}$ rRNA gene, as previously described [24]. Stool and blood samples were also examined to rule out other infective etiology for diarrhea symptoms. Accordingly, all stool samples were examined for Campylobacter spp., Shigella spp., Salmonella spp. Yersinia enterocolitica, and pathogenic E. coli strains by culture method and molecular assays, as previously described $[25,26]$. Moreover, blood samples of the patients were tested for anti- CMV antibodies of IgG and IgM subclasses using a commercially available ELISA kit according to the manufacturer's instructions (Vircell, Spain).

\section{Molecular assay for clostridial toxins}

DNA was extracted from the colonies grown on specific media using DNeasy Blood \& Tissue Kit (Qiagen, Hilden, Germany) according to the manufacturer's procedure. The quality and purity of the extracted DNA were measured using NanoDrop (Thermo Scientific, Asheville, NC, USA). The presence of toxin genes of $C$. difficile was examined using PCR by specific primers, as previously described $[27,28]$.

\section{Procalcitonin and CRP measurement}

The serum procalcitonin level was measured in patients with IBD using VIDAS ${ }^{\circledR}$ B.R.A.H.M.S PCT ${ }^{\mathrm{TM}}$ Assay (bioMérieux Inc., Durham, USA) according to the manufacturer's instructions. The normal range of serum procalcitonin levels was $0-0.5 \mathrm{ng} / \mathrm{mL}$. The serum CRP levels were determined by using commercially available assay kits (Roche Diagnostics GmbH, Mannheim, Germany). The normal serum CRP level was considered to be $<0.8 \mathrm{mg} / \mathrm{dL}$.

\section{Statistical analysis}

Data were presented as median (interquartile range [IQR]) or $\mathrm{n}(\%)$. Comparisons between groups (CDI + and CDI- groups) were performed by Mann-Whitney or T-test for continuous variables according to data distribution. For the diagnostic evaluation of procalcitonin, the sensitivity and specificity for the calculated cut-off point were recorded. Receiver operating characteristic (ROC) curve analysis was conducted to determine the diagnostic performance of procalcitonin in detecting CDI among
IBD patients. Accordingly, we estimated the area under the curve (AUC) for procalcitonin assay compared to the culture of $C$. difficile in four fitted models, separately. In model 1, we used univariate logistic regression to test if $\log 10$ procalcitonin was significantly associated with a $\mathrm{CDI}+$. In Model 2, we used multiple logistic regression with the following additional risk factors to see if they were associated with a CDI+: age, hospitalization, type of IBD (CD or UC), duration of the disease, frequency of defecation per day, and antibiotic usage. In Model 3, we reconstructed a multiple logistic regression with CRP and ESR to examine how these biomarkers interacted with procalcitonin. The final multivariable model was fitted for $\log 10$ procalcitonin based on the phase of IBD (i.e., flare or remission) (Model 4). The diagnostic performance of our models was assessed by R software version 3.6, package ROCR [29]. Other statistical analyses were performed using SPSS software version 22.0 (Chicago, IL, USA). A $P$-value $<0.05$ was considered to indicate statistical significance.

\section{Results}

Baseline demographics and clinical characteristics

One hundred twenty IBD patients (mean $36.08 \pm 14.37$ years; 67 females and 53 males) consisting of 111 (92.5\%) UC and 9 (7.5\%) CD were included in the study. The demographic and clinical characteristics of the patients are summarized in Table 1 . IBD remission was observed in $23.4 \%(26 / 111)$ of UC and $44.4 \%(4 / 9)$ of $\mathrm{CD}$ patients, while flare phase was found in $76.6 \%$ of UC $(85 / 111)$ and $55.5 \%$ of CD (5/9) patients. The extent of disease involvement in UC patients included pancolitis $9 \%(10 / 111)$, extensive colitis $3.6 \%$ (4/111), left-sided colitis $10.8 \%(12 / 111)$, proctitis $60.4 \%(67 / 111)$, backwash ileitis 16.2\% (18/111), Crohn colitis 55.5\% (5/9) and Crohn ileitis $44.4 \%(4 / 9)$.

\section{Microbial assessment}

Totally, 17 (14.2\%) patients with IBD were CDI positive, in which $34 \mathrm{C}$. difficile isolates were recovered simultaneously from stool and biopsy samples of these patients. $C$. difficile was isolated from $15.3 \%$ (13/85) of UC patients with flare and $11.5 \%(3 / 26)$ in remission. Only one isolate (20\%) of C. difficile was isolated from a CD patient with the flare of the disease. All $C$. difficile isolates harbored genes encoding toxins $\mathrm{A}$ and $\mathrm{B}$.

\section{Serum procalcitonin, CRP, and ESR levels in CDI and non-CDI groups}

Serum PCT, CRP, and ESR levels concerning to CDI or non-CDI, as well as flare or remission are shown in Fig. 1. The median (IQR) serum procalcitonin levels in CDI group and non-CDI group were $0.69 \mathrm{ng} / \mathrm{mL}$ 
Table 1 Demographic data and clinical characteristics of the IBD patients

\begin{tabular}{|c|c|c|c|c|}
\hline \multirow[t]{2}{*}{ Characteristics } & \multicolumn{2}{|l|}{ UC $(n=111)$} & \multicolumn{2}{|l|}{$\mathrm{CD}(n=9)$} \\
\hline & Flare $(n=85)$ & Remission $(n=26)$ & Flare $(n=5)$ & Remission $(n=4)$ \\
\hline \multicolumn{5}{|l|}{ Gender } \\
\hline Female & $50(58.8)$ & $14(53.8)$ & $3(60)$ & 0 \\
\hline Male & $35(41.2)$ & $12(46.2)$ & $2(40)$ & $4(100)$ \\
\hline \multicolumn{5}{|l|}{ Age } \\
\hline $1-20$ & $4(4.7)$ & $5(19.2)$ & 0 & 0 \\
\hline $21-30$ & $28(32.9)$ & $11(42.3)$ & $1(20)$ & $3(75)$ \\
\hline $31-40$ & $21(24.7)$ & $3(11.5)$ & $1(20)$ & 0 \\
\hline $41-50$ & $18(21.2)$ & $2(7.7)$ & $2(40)$ & 0 \\
\hline $51-60$ & $5(5.9)$ & $4(15.4)$ & 0 & $1(25)$ \\
\hline $61-70$ & $5(5.9)$ & $1(3.9)$ & $1(20)$ & 0 \\
\hline $71-80$ & $4(4.7)$ & 0 & 0 & 0 \\
\hline \multicolumn{5}{|l|}{ Extent of disease } \\
\hline Pancolitis & $7(8.2)$ & $3(11.5)$ & 0 & 0 \\
\hline Extensive colitis & $4(4.7)$ & 0 & 0 & 0 \\
\hline Left-sided colitis & $10(11.8)$ & $2(7.7)$ & 0 & 0 \\
\hline Proctitis & $55(64.7)$ & $12(46.2)$ & 0 & 0 \\
\hline Backwash ileitis & $13(15.3)$ & $5(19.2)$ & 0 & 0 \\
\hline Crohn colitis & 0 & 0 & $1(20)$ & $4(100)$ \\
\hline Crohn ileitis & 0 & 0 & $4(80)$ & 0 \\
\hline \multicolumn{5}{|l|}{ Consistency of stool } \\
\hline Watery & $51(60)$ & $6(23.1)$ & $4(80)$ & $2(50)$ \\
\hline Loosed & $34(40)$ & $20(76.9)$ & $1(20)$ & $2(50)$ \\
\hline Bloody & $39(45.9)$ & 0 & $2(40)$ & $1(25)$ \\
\hline \multicolumn{5}{|l|}{ Clinical manifestation } \\
\hline Anorexia & $14(16.5)$ & $3(11.5)$ & 0 & $1(25)$ \\
\hline Abdominal tenderness & $22(25.9)$ & $4(15.4)$ & $3(60)$ & $2(50)$ \\
\hline Nausea and vomiting & $23(27.1)$ & $9(34.6)$ & $3(60)$ & $2(50)$ \\
\hline
\end{tabular}

IBD inflammatory bowel disease, UC Ulcerative colitis, $C D$ Crohn's disease

(0.5-0.98 ng/mL) and $0.32 \mathrm{ng} / \mathrm{mL}(0.03-0.67 \mathrm{ng} / \mathrm{mL})$, respectively. Serum procalcitonin level was significantly increased in IBD patients with CDI compared to nonCDI IBD patients $(P$-value $<0.0001)$, however, this difference was not observed between IBD patients with and without a flare (Fig. 1A vs D). The median (IQR) serum CRP level in IBD patients with CDI was also higher than that for patients without CDI, $14.82 \mathrm{mg} / \mathrm{dL}(13.2-$ $16.3 \mathrm{mg} / \mathrm{dL})$ vs $10.06 \mathrm{mg} / \mathrm{dL}(9.4-10.5 \mathrm{mg} / \mathrm{dL})$, respectively, $(P$-value $<0.001)$. Moreover, ESR level in patients with CDI displayed significantly increased $(21.24 \mathrm{~mm} / \mathrm{h}$ [18.12-23.83 $\mathrm{mm} / \mathrm{h}])$ compared to non-CDI group $(11.41 \mathrm{~mm} / \mathrm{h}[10.77-11.64 \mathrm{~mm} / \mathrm{h}])(P$-value $<0.0001)$.

\section{ROC curve, sensitivity, and specificity}

By univariate logistic regression, $\log _{10}$ procalcitonin was associated with CDI (OR 2.81, 95\% CI 1.54-4.09, $P$-value $<0.001)$ (Fig. 2). In the ROC curve analyses, the AUC for procalcitonin was 0.89 (95\% CI 0.82-0.95). The best cut-off value for procalcitonin to diagnose CDI was $1.1 \mathrm{ng} / \mathrm{mL}$; this threshold value was associated with sensitivity and specificity of $85 \%$ and $88 \%$, respectively. In Model 2, which included $\log _{10}$ procalcitonin, age, hospitalization, type of IBD (CD or UC), duration of the disease, frequency of defecation per day, and antibiotic usage, procalcitonin showed a robust association with CDI (OR 4.59, 95\% CI 2.49-6.70, $P$-value < 0.001) (Fig. 2, Table 2). The AUC of Model 2 was increased to 0.92 (95\% CI $0.86-0.97$ ), the sensitivity was $88 \%$ and the specificity was $88 \%$. In Model 3 , which included $\log _{10}$ procalcitonin, CRP, and ESR, procalcitonin continued to show a strong association with CDI (OR 2.85, 95\% CI 1.55-4.16, $P$-value $<0.001$ ) (Fig. 2, Table 2). However, the AUC was decreased to 0.7 (95\% CI 0.56-0.83), which was associated with a sensitivity of $56 \%$ and specificity of $88 \%$. Furthermore, $\log _{10}$ procalcitonin remained a significant predictor of CDI after adjusting for the flare phase of the disease, Model 4, (OR 2.81, 95\% CI 1.53-4.09, 

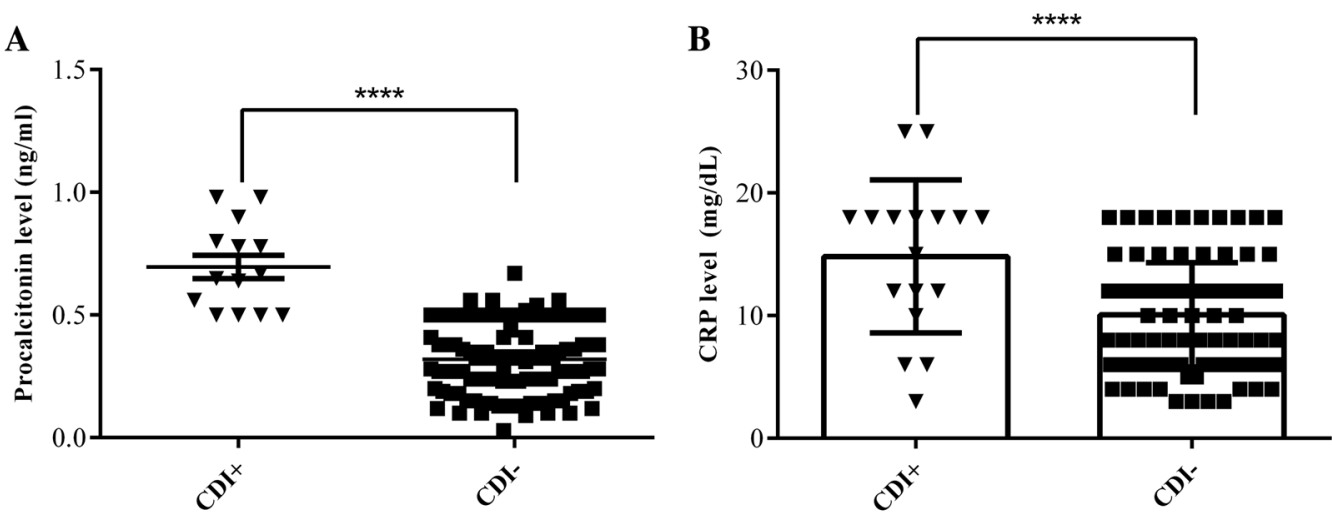

$\mathbf{C}$
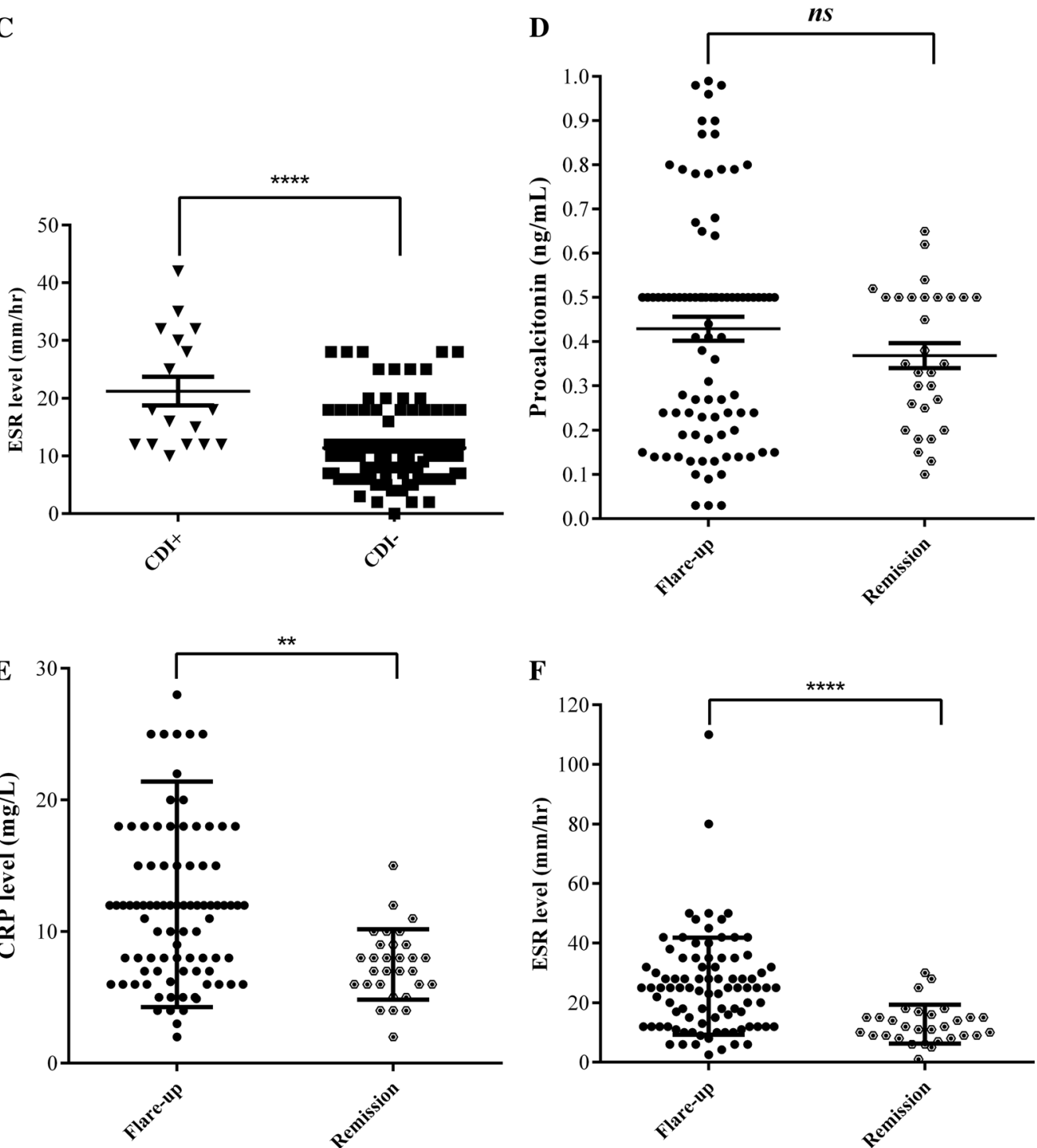

Fig. 1 Scattergrams of serum procalcitonin levels (A), CRP (B), and ESR (C) in IBD patients with CDI $(n=17)$ and IBD patients without CDI ( $n=103)$. Graphs (D-F) represent the levels of procalcitonin, CRP, and ESR in IBD patients with flare $(n=30)$ compared to IBD patients in remission ( $n=90)$, respectively. Error bars represent the median interquartile range (IQR). The student's t-test was used for data analysis. ${ }^{* *},{ }^{* * * *}$ and $n s$ denote $P$-value $<0.01, P$-value $<0.0001$ and non-significant, respectively 


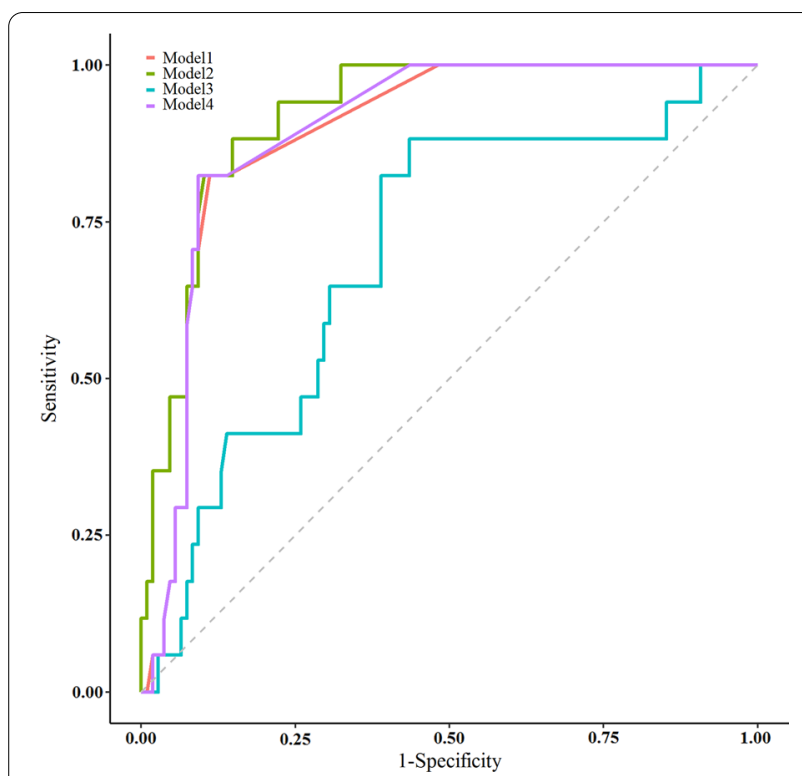

Fig. 2 Receiver operating characteristic (ROC) curves for procalcitonin were reconstructed in 4 models. In Model 1, the estimated area under the curve (AUC) for procalcitonin was calculated at 0.89 ( $95 \% \mathrm{Cl} 0.82-0.95)$ by univariate logistic regression. The AUC of Model 2 was increased to 0.92 (95\% Cl 0.86-0.97), the sensitivity was $88 \%$ and the specificity was $88 \%$. In Model 3 , the AUC was decreased to 0.7 (95\% Cl 0.56-0.83), which was associated with a sensitivity of $56 \%$ and specificity of $88 \%$. Finally, Model 4 revealed that the AUC was 0.89 (95\% Cl $0.83-0.95)$ by the sensitivity of $85 \%$ and specificity of $90 \%$

Table 2 Odds ratios for predictors of CDI in IBD patients based on univariate (Model 1) and multiple (Models 2-4) logistic regression

\begin{tabular}{|c|c|c|c|}
\hline Variables & Odds ratio & [95\% Conf. Interval] & $P$-value \\
\hline \multicolumn{4}{|l|}{ Model 1} \\
\hline Procalcitonin & 2.81 & $1.54-4.09$ & $<0.001$ \\
\hline \multicolumn{4}{|l|}{ Model 2} \\
\hline Procalcitonin & 4.59 & $2.49-6.70$ & $<0.001$ \\
\hline Age & 1.01 & $0.97-1.06$ & 0.45 \\
\hline UC & 0.11 & $0.01-0.97$ & 0.04 \\
\hline$C D$ & 9.09 & $2.01-53.97$ & $<0.001$ \\
\hline Hospitalization & 0.20 & $0.00-5.04$ & 0.33 \\
\hline IBD duration & 0.91 & $0.79-1.05$ & 0.21 \\
\hline Antibiotic usage & 6.87 & $1.12-41.98$ & 0.03 \\
\hline \multicolumn{4}{|l|}{ Model 3} \\
\hline Procalcitonin & 2.85 & $1.55-4.16$ & $<0.001$ \\
\hline CRP & 0.93 & $0.80-1.09$ & 0.41 \\
\hline ESR & 0.98 & $0.98-1.09$ & 0.11 \\
\hline \multicolumn{4}{|l|}{ Model 4} \\
\hline Procalcitonin & 2.81 & $1.53-4.09$ & $<0.001$ \\
\hline Flare & 5.08 & $0.00-0.45$ & 0.007 \\
\hline
\end{tabular}

Bold values denote a statistically significant result at the $P$-value $<0.05$ $C D I$ Clostridioides difficile infection, IBD inflammatory bowel disease, UC Ulcerative colitis, CD Crohn's disease, CRP C-reactive protein, ESR Erythrocyte sedimentation rate level
$P$-value $<0.001$ ) (Fig. 2, Table 2). Accordingly, our analysis revealed that the AUC of adjusted procalcitonin and flare phase of the disease in predicting CDI was 0.89 (95\% CI $0.83-0.95$ ) by the sensitivity of $85 \%$ and specificity of $90 \%$.

\section{Discussion}

Clinical presentation and laboratory data between disease flare and CDI are often similar among patients with IBD. It is clinically challenging to early differentiate CDI from disease flare because treatment strategies according to the disease course are completely different. Importantly, in the case of CDI, early detection and appropriate therapy can lead to a good prognosis in patients with IBD [30, 31]. Moreover, the culture of $C$. difficile is the most sensitive test but is labor-intensive and results may be delayed up to 4-7 days. Although Rao and colleagues previously described that an elevated procalcitonin level is associated with severe CDI and CDI is unlikely to be severe with a serum procalcitonin level below $0.2 \mathrm{ng} /$ $\mathrm{mL}$ [32], there is limited data available for the diagnostic power of procalcitonin to detect CDI in patients with IBD. Several studies have previously proposed that serum procalcitonin could be a useful biomarker for early diagnosis of sepsis [33], respiratory infections [34], postoperative infections [35], ventilator-associated pneumonia [36], and severe CDI [32]. To the best of our knowledge, this is the first study conducted to evaluate the diagnostic value of procalcitonin in early diagnosis of CDI in IBD patients. Compared with culture and based on the best cut-off value $(1.1 \mathrm{ng} / \mathrm{mL})$ calculated in our study, serum procalcitonin had an acceptable specificity $(85 \%)$ and sensitivity (88\%) in the diagnosis of CDI.

Our data showed that serum procalcitonin, CRP, and ESR levels were significantly increased among IBD patients with CDI compared to those without CDI. All these biomarkers are measured as part of routine GI investigations, particularly for UC [37], however, some authors reported that CRP and ESR are limited by their inability to distinguish enteric infections from other inflammatory conditions [38-40]. Serum procalcitonin has been reported to offer better sensitivity and specificity than CRP and ESR for the exact diagnosis of bacterial infections [41, 42]. Our data showed that there was no significant association between serum procalcitonin level and flare of IBD regardless of CDI and serum procalcitonin was only increased in flares in patients with CDI. In the line of these findings, Chung and colleagues have described that serum procalcitonin level is not affected by IBD activity itself, although they may be affected by concomitant enteric infections and is more useful than CRP to distinguish infection stage from a flare-up of the disease [43]. 
Though we hypothesized that serum procalcitonin level would associate with other factors such as prolonged antibiotic courses, stage of disease, IBD duration, and type of IBD (CD or UC). We conducted multivariate analyses adjusted with procalcitonin to determine the exact relationship between this biomarker and CDI. Importantly, determination of previous antibiotic usage, type of IBD, and stage of the disease could be adjusted to maximize the prediction of CDI in patients with IBD. Although there existed a strong association between procalcitonin measurement and CDI diagnosis in the adjusted model with CRP and ESR, the sensitivity of the test was dramatically decreased. Additionally, according to our analysis (Model 4), procalcitonin measurement in IBD patients during the flare-up phase more robustly predicts CDI, thus, it may be applied for diagnosis and management of CDI in this group of patients.

This study was limited by enrolling a relatively small number of IBD patients with underlying CDI. In particular, the number of IBD patients with concomitant CDI was not equal to IBD patients without CDI. Second, the subjects were enrolled retrospectively from a single tertiary center, which may have caused an unavoidable selection bias. Furthermore, we could not follow up the subjects to evaluate the procalcitonin level before and after antibiotic treatments to know the relationship between the change in procalcitonin levels and treatment of CDI. Finally, we did not evaluate co-infection with other enteric bacterial or viral agents to whether procalcitonin could discriminate between CDI and other enteric co-infections.

\section{Conclusion}

Our study indicated that the measurement of serum procalcitonin level can be useful in the early detection of CDI complications in IBD patients. A higher serum procalcitonin level may reflect the CDI in the stage of the flare-up. Therefore, serum procalcitonin level can be a candidate biomarker for assessing the CDI in IBD patients. However, future large-scale investigations should be performed to validate our results, to extend them to understand whether procalcitonin level measured at the flare-up stage predict CDI, and to define the role of this biomarker in treatment algorithms for CDI.

\footnotetext{
Abbreviations

CDI: Clostridioides difficile Infection; Clostridioides difficile: C. difficile; IBD: Inflammatory bowel disease; ROC: Receiver operating characteristic; CD: Crohn's disease; UC: Ulcerative colitis; GI: Gastrointestinal; ESR: Erythrocyte sedimentation rate; WBCs: White blood cells; CRP: C-reactive protein; CDAl: Crohn's disease activity index; CCFA: Cycloserine-cefoxitin-fructose agar.
}

\section{Acknowledgements}

The authors wish to thank the Taleghani Hospital Endoscopy Department for their cooperation and help performing this study. We also are grateful to all laboratory staff of the Foodborne and Waterborne Diseases Research Center, Research Institute for Gastroenterology and Liver Diseases, Shahid Beheshti University of Medical Sciences, Tehran, Iran.

\section{Authors' contributions}

MA contributed to colonoscopy procedures and data collection; MAz performed microbiological experiments; $\mathrm{HH}$ and MAz wrote the manuscript draft; BN and FA performed sample collection and microbiological experiments; MO performed the statistical analysis; OKNS participated in patients' clinical data collection; AY and SS contributed to study design, methodology, conceptualization and project administration; AY, SS, MMR, HAA and MRZ provided important intellectual content and critically revised the manuscript. All authors read and approved the final manuscript.

\section{Funding}

The study was supported by a research grant (Project No: RIGLD 1063) from the Foodborne and Waterborne Diseases Research Center, Research Institute for Gastroenterology and Liver Diseases, Shahid Beheshti University of Medical Sciences, Tehran, Iran. The funding bodies had no role in study design, data collection, data analysis, decision to publish, or preparation of the manuscript.

\section{Availability of data and materials}

The datasets used and/or analyzed during the current study available from the corresponding author on reasonable request.

\section{Declarations}

Ethics approval and consent to participate

This study was approved by the Institutional Ethical Review Committee of Research Institute for Gastroenterology and Liver Diseases at Shahid Beheshti University of Medical Sciences (Project No. IR.SBMU.RIGLD.REC.1398.041). All experiments were performed in accordance with the relevant guidelines and regulations recommended by the Research Institute for Gastroenterology and Liver Diseases. Informed consent was obtained from all subjects and/or their legal guardians before sample collection.

\section{Consent for publication}

Not applicable.

\section{Competing interests}

The authors declare no competing interests.

\section{Author details}

${ }^{1}$ Gastroenterology and Liver Diseases Research Center, Research Institute for Gastroenterology and Liver Diseases, Shahid Beheshti University of Medical Sciences, Tehran, Iran. ${ }^{2}$ Foodborne and Waterborne Diseases Research Center, Research Institute for Gastroenterology and Liver Diseases, Shahid Beheshti University of Medical Sciences, Tehran, Iran. ${ }^{3}$ Basic and Molecular Epidemiology of Gastrointestinal Disorders Research Center, Research Institute for Gastroenterology and Liver Diseases, Shahid Beheshti University of Medical Sciences, Tehran, Iran.

Received: 10 July 2021 Accepted: 20 October 2021

Published online: 26 October 2021

\section{References}

1. Wilson JC, Furlano RI, Jick SS, Meier CR. Inflammatory bowel disease and the risk of autoimmune diseases. J Crohn's Colitis. 2016;10(2):186-93.

2. Clooney AG, Eckenberger J, Laserna-Mendieta E, Sexton KA, Bernstein MT, Vagianos K, Sargent M, Ryan FJ, Moran C, Sheehan D et al. Ranking microbiome variance in inflammatory bowel disease: a large longitudinal intercontinental study. Gut 2020;gutjnl-2020-321106.

3. Ananthakrishnan AN. Epidemiology and risk factors for IBD. Nat Rev Gastroenterol Hepatol. 2015;12(4):205-17. 
4. Cosnes J, Gower-Rousseau C, Seksik P, Cortot A. Epidemiology and natural history of inflammatory bowel diseases. Gastroenterology. 2011;140(6):1785-94.

5. Sartor RB. Microbial influences in inflammatory bowel diseases. Gastroenterology. 2008;134(2):577-94.

6. Axelrad JE, Joelson A, Green PHR, Lawlor G, Lichtiger S, Cadwell K, Lebwohl B. Enteric infections are common in patients with flares of inflammatory bowel disease. Am J Gastroenterol. 2018;113(10):1530-9.

7. Mann EA, Saeed SA. Gastrointestinal infection as a trigger for inflammatory bowel disease. Curr Opin Gastroenterol. 2012;28(1):24-9.

8. Epple HJ. Therapy- and non-therapy-dependent infectious complications in inflammatory bowel disease. Dig Dis. 2009;27(4):555-9.

9. Kucharzik T, Maaser C. Infections and chronic inflammatory bowel disease. Viszeralmedizin. 2014;30(5):326-32.

10. Navaneethan $U$, Venkatesh PG, Shen B. Clostridium difficile infection and inflammatory bowel disease: understanding the evolving relationship. World J Gastroenterol. 2010;16(39):4892-904.

11. Gholam-Mostafaei FS, Yadegar A, Aghdaei HA, Azimirad M, Daryani NE, Zali MR. Anti-TNF containing regimens may be associated with increased risk of Clostridioides difficile infection in patients with underlying inflammatory bowel disease. Curr Res Transl Med. 2020;68(3):125-30.

12. Kodori M, Ghalavand Z, Yadegar A, Eslami G, Azimirad M, Krutova M, Abadi A, Zali MR. Molecular characterization of pathogenicity locus (PaLoc) and tcdC genetic diversity among tcdA(+)B(+)Clostridioides difficile clinical isolates in Tehran, Iran. Anaerobe. 2020;66:102294.

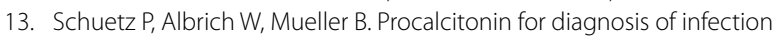
and guide to antibiotic decisions: past, present and future. BMC Med. 2011;9(1):107

14. Yang DH, Yang SK, Park SH, Lee HS, Boo SJ, Park JH, Na SY, Jung KW, Kim $\mathrm{KJ}$, Ye BD, et al. Usefulness of C-reactive protein as a disease activity marker in Crohn's disease according to the location of disease. Gut Liver. 2015;9(1):80-6.

15. Lee H. Procalcitonin as a biomarker of infectious diseases. Korean J Intern Med. 2013;28(3):285-91.

16. Lippi G, Sanchis-Gomar F. Procalcitonin in inflammatory bowel disease: drawbacks and opportunities. World J Gastroenterol. 2017;23(47):8283-90.

17. Kim S-E. Serum procalcitonin is a candidate biomarker to differentiate bacteremia from disease flares in patients with inflammatory bowel disease. Gut Liver. 2016;10(4):491-2.

18. Däbritz J, Langhorst J, Lügering A, Heidemann J, Mohr M, Wittkowski $H$, Krummenerl T, Foell D. Improving relapse prediction in inflammatory bowel disease by neutrophil-derived S100A12. Inflamm Bowel Dis. 2013;19(6):1130-8.

19. Kessel C, Lavric M, Weinhage T, Brueckner M, de Roock S, Däbritz J, Weber J, Vastert SJ, Foell D. Serum biomarkers confirming stable remission in inflammatory bowel disease. Sci Rep. 2021;11(1):6690.

20. Travis SP, Higgins PD, Orchard T, Van Der Woude CJ, Panaccione R, Bitton A, O'Morain C, Panés J, Sturm A, Reinisch W, et al. Review article: defining remission in ulcerative colitis. Aliment Pharmacol Ther. 2011;34(2):113-24.

21. Juncadella A, Papamichael K, Vaughn BP, Cheifetz AS. Maintenance adalimumab concentrations are associated with biochemical, endoscopic, and histologic remission in inflammatory bowel disease. Dig Dis Sci. 2018;63(11):3067-73.

22. Scaioli E, Cardamone C, Scagliarini M, Zagari RM, Bazzoli F, Belluzzi A Can fecal calprotectin better stratify Crohn's disease activity index? Ann Gastroenterol. 2015;28(2):247-52.

23. McDonald LC, Coignard B, Dubberke E, Song X, Horan T, Kutty PK. Recommendations for surveillance of Clostridium difficile-associated disease. Infect Control Hospital Epidemiol. 2007;28(2):140-5.

24. Azimirad M, Krutova M, Yadegar A, Shahrokh S, Olfatifar M, Aghdaei HA, Fawley WN, Wilcox MH, Zali MR. Clostridioides difficile ribotypes 001 and 126 were predominant in Tehran healthcare settings from 2004 to 2018: a 14-year-long cross-sectional study. Emerging Microbes Infect 2020:1-39.

25. Cunningham SA, Sloan LM, Nyre LM, Vetter EA, Mandrekar J, Patel R. Three-hour molecular detection of Campylobacter, Salmonella, Yersinia, and Shigella species in feces with accuracy as high as that of culture. J Clin Microbiol. 2010;48(8):2929-33.
26. Razaghi M, Tajeddin E, Ganji L, Alebouyeh M, Alizadeh AHM, Sadeghi A, Zali MR. Colonization, resistance to bile, and virulence properties of Escherichia coli strains: unusual characteristics associated with biliary tract diseases. Microb Pathog. 2017;111:262-8.

27. Persson S, Torpdahl M, Olsen K. New multiplex PCR method for the detection of Clostridium difficile toxin $A(t c d A)$ and toxin $B(t c d B)$ and the binary toxin (cdtA/cdtB) genes applied to a Danish strain collection. Clin Microbiol Infect. 2008;14(11):1057-64.

28. Azimirad M, Krutova M, Balaii H, Kodori M, Shahrokh S, Azizi O, Yadegar A, Aghdaei HA, Zali MR. Coexistence of Clostridioides difficile and Staphylococcus aureus in gut of Iranian outpatients with underlying inflammatory bowel disease. Anaerobe. 2020;61:102113.

29. Sing T, Sander O, Beerenwinkel N, Lengauer T. ROCR: visualizing classifier performance in R. Bioinformatics. 2005;21(20):3940-1.

30. Rodemann JF, Dubberke ER, Reske KA, Seo DH, Stone CD. Incidence of Clostridium difficile infection in inflammatory bowel disease. Clin Gastroenterol Hepatol. 2007;5(3):339-44.

31. Issa M, Ananthakrishnan AN, Binion DG. Clostridium difficile and inflammatory bowel disease. Inflamm Bowel Dis. 2008;14(10):1432-42.

32. Rao K, Walk ST, Micic D, Chenoweth E, Deng L, Galecki AT, Jain R, Trivedi I, Yu M, Santhosh K. Procalcitonin levels associate with severity of Clostridium difficile infection. PLoS ONE. 2013;8(3):e58265.

33. Nobre V, Harbarth S, Graf J-D, Rohner P, Pugin J. Use of procalcitonin to shorten antibiotic treatment duration in septic patients: a randomized trial. Am J Respir Crit Care Med. 2008;177(5):498-505.

34. Schuetz P, Christ-Crain M, Thomann R, Falconnier C, Wolbers M, Widmer I, Neidert S, Fricker T, Blum C, Schild U. Effect of procalcitonin-based guidelines vs standard guidelines on antibiotic use in lower respiratory tract infections: the ProHOSP randomized controlled trial. JAMA. 2009;302(10):1059-66.

35. Hochreiter M, Köhler T, Schweiger AM, Keck FS, Bein B, von Spiegel T, Schroeder S. Procalcitonin to guide duration of antibiotic therapy in intensive care patients: a randomized prospective controlled trial. Crit Care. 2009;13(3):1-7.

36. Stolz D, Smyrnios N, Eggimann P, Pargger H, Thakkar N, Siegemund M, Marsch S, Azzola A, Rakic J, Mueller B. Procalcitonin for reduced antibiotic exposure in ventilator-associated pneumonia: a randomised study. Eur Respir J. 2009;34(6):1364-75.

37. Tibble J, Teahon K, Thjodleifsson B, Roseth A, Sigthorsson G, Bridger S, Foster R, Sherwood R, Fagerhol M, Bjarnason I. A simple method for assessing intestinal inflammation in Crohn's disease. Gut. 2000;47(4):506-13.

38. Limper M, De Kruif M, Duits A, Brandjes D, Van Gorp E. The diagnostic role of procalcitonin and other biomarkers in discriminating infectious from non-infectious fever. J Infect. 2010;60(6):409-16.

39. Meynaar IA, Droog W, Batstra M, Vreede R, Herbrink P: In critically ill patients, serum procalcitonin is more useful in differentiating between sepsis and SIRS than CRP, II-6, or LBP. Crit Care Res Practice 2011, 2011.

40. Delevaux I, Andre M, Colombier M, Albuisson E, Meylheuc F, Bègue R, Piette J, Aumaître O. Can procalcitonin measurement help in differentiating between bacterial infection and other kinds of inflammatory processes? Ann Rheum Dis. 2003;62(4):337-40.

41. Floriańczyk B. Structure and diagnostic value of procalcitonin. Annales Universitatis Mariae Curie-Sklodowska Sectio D: Medicina. 2003;58(1):338-42.

42. Simon L, Gauvin F, Amre DK, Saint-Louis P, Lacroix J. Serum procalcitonin and C-reactive protein levels as markers of bacterial infection: a systematic review and meta-analysis. Clin Infect Dis. 2004;39(2):206-17.

43. Chung SH, Lee HW, Kim SW, Park SJ, Hong SP, Kim TI, Kim WH, Cheon $\mathrm{JH}$. Usefulness of measuring serum procalcitonin levels in patients with inflammatory bowel disease. Gut Liver. 2016;10(4):574.

\section{Publisher's Note}

Springer Nature remains neutral with regard to jurisdictional claims in published maps and institutional affiliations. 